\title{
The mutual interference vortex flow from a pair of in-line forced oscillating staggered arranged circular cylinders
}

\author{
Yoshifumi YOKOI* and Keiko HIRAO** \\ * Department of Mechanical Engineering, National Defense Academy of Japan \\ 1-10-20 Hashirimizu, Yokosuka-shi, Kanagawa 239-8686, Japan \\ E-mail: yokoi@nda.ac.jp \\ ** Iruma Base, Japan Air Self-Defense Force \\ 2-3 Inariyama, Sayama-shi, Saitama 350-1394, Japan
}

Received 28 January 2014

\begin{abstract}
In order to understand the aspect of the mutual interference flow from two circular cylinders, the visual observation experiment was performed. The cylinder setting conditions were three kinds of distance ratios $(L / d=1.5,2.5$ and 5.5), and seven kinds of arrangement angles $(\alpha=0,15,30,45,60,75$ and 90 degrees). The oscillating conditions were four kinds of amplitude ratios $(2 a / d=0.25,0.5,0.75$ and 1.0$)$, and the oscillation frequency ratio $f / f_{K}$ in 24 steps. The Reynolds number was about 640 . As the result of experiment, even if the distance ratio was the same, the vortex shedding characteristics changed with arrangement angles. The mutual interference will become remarkable if the distance ratio is small. In the arrangement angle, 30 degrees and 45 degrees are carrying out mutual interference most. Even when a forced in-line oscillation was performed under the conditions in which two circular cylinders are carrying out mutual interference, it was found that a lock-in phenomenon occurs. The vortex shedding features were obtained and flow pattern distributions were shown. The lock-in characteristics were investigated and the lock-in ranges have been presented in each distance ratio. Four kinds of typical flow patterns at the time of the lock-in of staggered arrangement oscillating two circular cylinders were shown.
\end{abstract}

Key words : Vortex, Interference flow, Lock-in, Two cylinders, Flow visualization, Staggered arrange

\section{Introduction}

In order to understand the surrounding flow of the bluff cylinder placed into the flow, many experiments which used the circular cylinder have been performed until now. Many obtained knowledge is also accumulated. At the industrial flow field, since two or more objects exist in the flow in many cases, the reports about the interference flow of two circular cylinders which considered the situation are also accumulated (Zdravkovich, 1997, 2003). As study on the interference problem of two circular cylinders, there are many examples of study of in-line arrangement or parallel arrangement. However, there is scarcely an example of study of arrangement of the middle angle. Sumner et al. (2000) identified various flow patterns observed in the flow around two stationary cylinders arranged in a staggered configuration, and discussed the relation between the flow patterns and the vortex shedding frequencies in detail. Sakamoto et al. (2004a) investigated the time averaged fluctuating fluid forces of two identical circular cylinders in staggered configurations by wind tunnel experiment. Moreover, Sakamoto et al. (2004b) showed the characteristics of vortex shedding of two staggered arranged circular cylinders by wind tunnel experiment. And they present the new findings for vortex shedding frequencies of two cylinders. Kim and Sakamoto (2007) investigated on the characteristics of flow-induced vibrations of two circular cylinders in staggered arrangement by wind tunnel test and flow visual water tank test. As study of the vortex shedding in an unsteady flow, Sarpkaya and Cinar (1980) varied the attack angle $(\alpha=0$, 30,60 and 90 degrees) and distance ratio of two circular cylinders $(L / d=1.5 \sim 3.5)$, and performed investigation of fluid force which acts on each circular cylinder using an up-righted $U$ type oscillating water tank. Williamson (1985) 
performed measurement of flow visualization and pressure simultaneously, and investigated the relationship between the vortex shedding from two circular cylinders, and fluid force. As a result, it was explained that the variation of a lift and the force between circular cylinders change with cylinder intervals. Chan (1986) investigated in the flow the self oscillation of two circular cylinders arranged tandem, parallel and staggered, and surveyed the general formula to an oscillating response. Since the state where the steady flow was maintained is in a rare state industrially, accumulation of the data of an unsteady flow is desired.

The purpose of this study is accumulation of the basic data for comparison with the flow under unsteady flow. In this report, the interval and arrangement angle of two circular cylinders were varied, the visualization experiment was performed, and the vortex shedding characteristics and the flow pattern in each case were investigated. In consideration of the result obtained from the experiment, the vortex shedding characteristic from staggered arrangement two circular cylinders in the unsteady flow supposing the periodic variation of the relative flow velocity is reported.

\section{Experimental apparatus and method}

\subsection{Experimental apparatus}

The experimental apparatus consists of a closed circuit water channel, a cylinder oscillator, a set of visual apparatus and a set of data record equipment. Figure 1 shows the drawing of the closed circuit water channel. The closed circuit water channel is a vertical circulation type of $5.8 \mathrm{~m}$ in length, $1.2 \mathrm{~m}$ in width, and $2.5 \mathrm{~m}$ in height, and the volume of water is 4 tons. The water channel is consisted of water flow generation equipment ( 2 axial flow type pumps), rectification device, test section and 4 corner parts with guide vanes. The test section is $2 \mathrm{~m}$ in length, $0.8 \mathrm{~m}$ in width, $0.4 \mathrm{~m}$ in depth, and the flume structure with the surface of the water. In the test section, the window made of the glass of $1.5 \mathrm{~m}$ in length and $0.4 \mathrm{~m}$ in width has been installed in the both sides wall and the bottom for the observation. The velocity distribution in the test section was $\pm 1.5 \%$ against main flow velocity $0.4 \mathrm{~m} / \mathrm{s}$ from depth $50 \sim 350 \mathrm{~mm}$ and the test section center in the direction of width within the range of $\pm 300 \mathrm{~mm}$. There is a set of rail orbit on the flume flange and a carriage is set up. The oscillator which installed CCD (charge-coupled device) camera and test cylinder, the visualization apparatus are loaded into the carriage. The cylinder oscillator is using a Scotch-yoke mechanism and the oscillator consists of a small-sized variable motor with a controller, a turn disk and a connecting rod. The schematic diagram of the cylinder oscillator is shown in Fig. 2. The amplitude of oscillation is set by changing the rotation radius of connecting edge on the turn disk, while the frequency of oscillation is controlled by changing the revolution speed of the turn disk. The visualization apparatus consists of a CCD camera, a video recorder, a video monitor, an argon gas laser unit and two 500W halogen ramps.

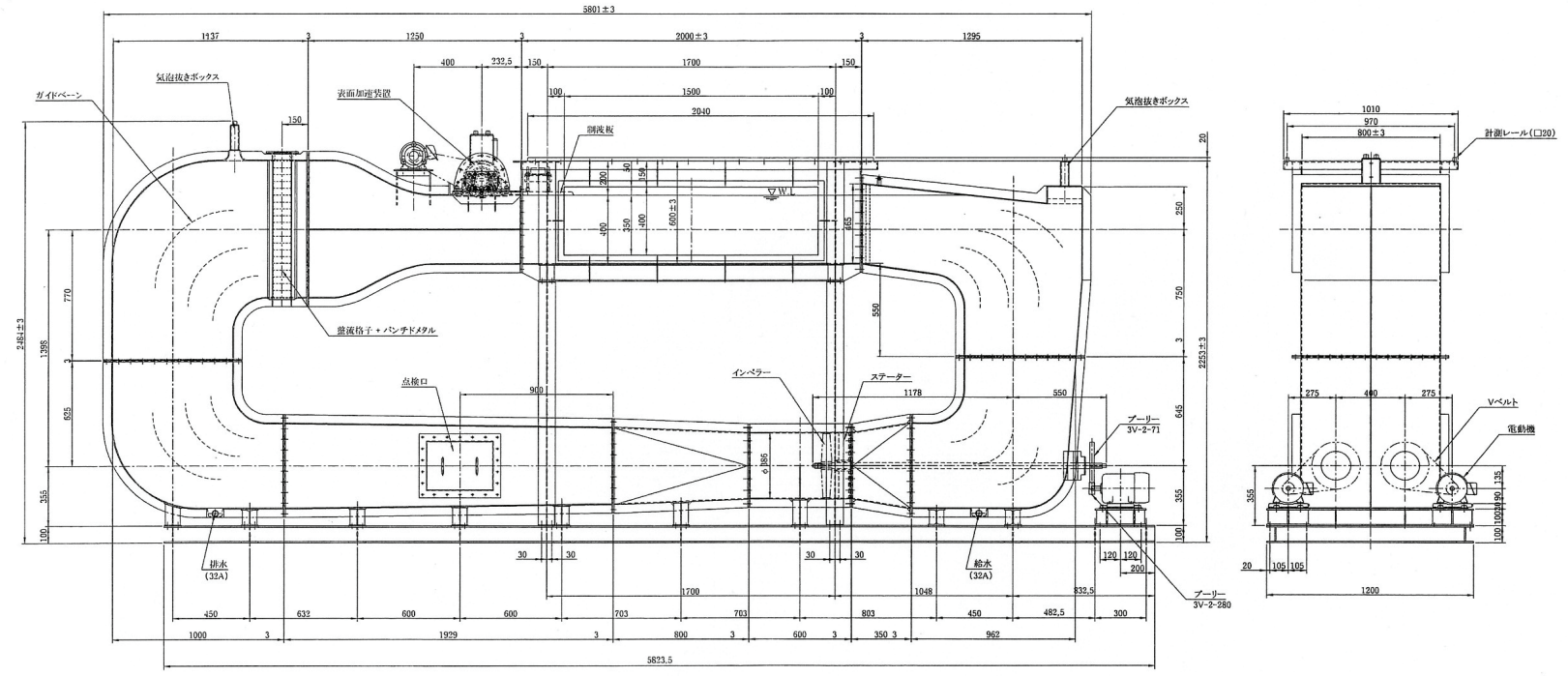

Fig. 1 Schematic figure of closed circuit water channel equipment. The carriage and some equipment on the carriage (oscillator, test cylinders and visualization equipments) are not written here. 


\subsection{Test cylinder}

The test circular cylinders are made from hollow aluminum with $16 \mathrm{~mm}$ of outside diameter, $14 \mathrm{~mm}$ caliber, and $600 \mathrm{~mm}$ length. Two cylinders of them without end plates are mounted vertically in a free-surface water channel, where its bottom end is free and its top end is clamped to a slide plate of cylinder oscillator. The appearance of the circular cylinders attached in the cylinder oscillator is shown in Fig. 3. Thus two circular cylinders are simultaneously given a longitudinal sinusoidal oscillation. The arrangement of circular cylinders is shown in Fig. 4. For convenience, front side and rear side cylinders are called the "1st cylinder" and "2nd cylinder", respectively. In parallel setting, the circular cylinders which located in left-hand side and right-hand side to the main flow were called the "left cylinder" and the "right cylinder", respectively.

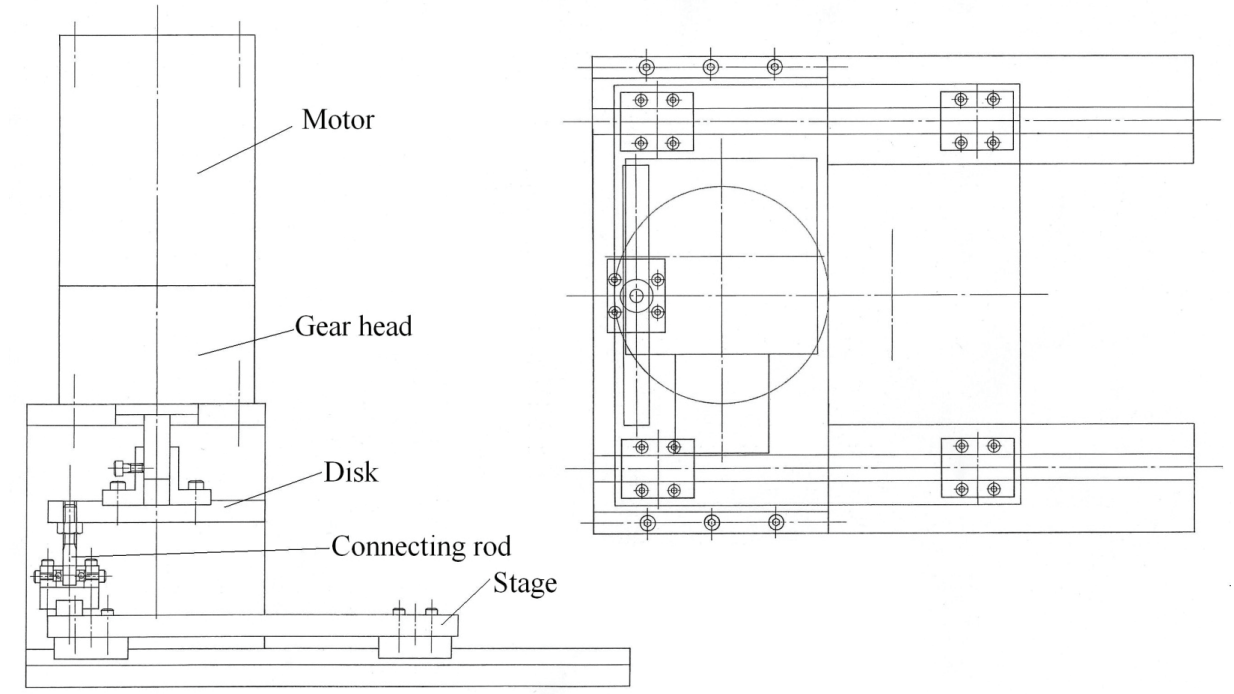

Fig. 2 The schematic diagram of the cylinder oscillator. It drives by a small motor, it slows down by a gear head, and a disk is rotated. It is changed into a straight-line motion from rotational movement by the slider rails arranged right-angled. Thereby, a stage moves reciprocate. Circular cylinders are attached in the stage.

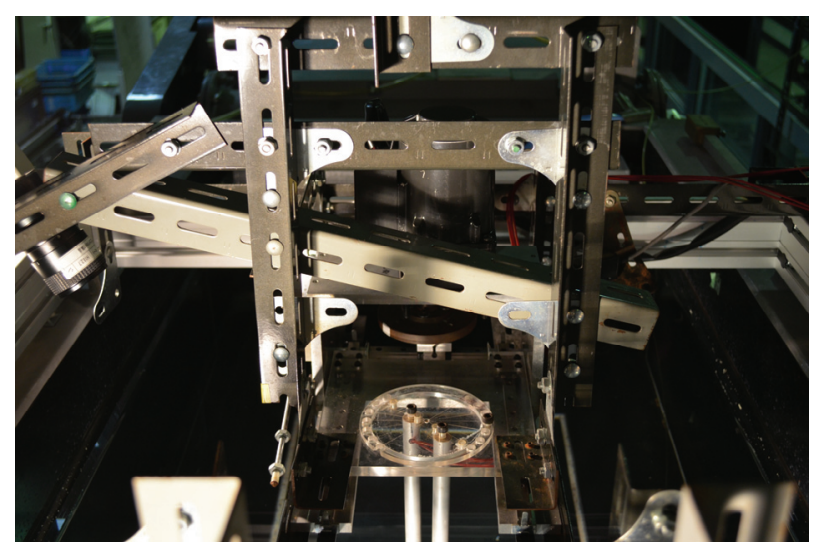

Fig. 3 The arrangement photograph of circular cylinders. This photograph is taken in bird's-eye view from the behind of a test section. Circular cylinders are attached in a slide stage. The vinyl tubes for tracer introduction are showing in a part for a cylinder upper end. The camera for observation is installed in the slanting upper part beside circular cylinders.

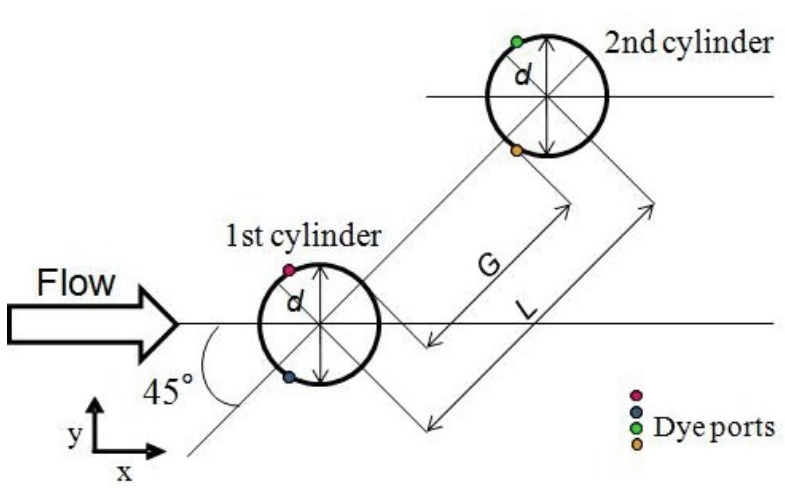

Fig. 4 Coordinate system and definition of symbols. The small circles with a color show the position where a tracer oozes. Each tracer oozing position is a position of 60 degrees from a front stagnation point. Here, $L=G+d$. 


\subsection{Flow visualization method}

A schematic diagram of a layout of flow visual apparatus is shown in Fig. 5. The horizontal section of the flow at a depth of $140 \mathrm{~mm}$ below the water surface which was enough for observation was visualized by the laser light sheet technique which was based on the dye injection method. The visualized flow patterns were monitored by the CCD video camera which set above the surface of the water. In this experiment, an argon gas laser beam (4W maximum power) was conducted to a cylindrical lens, which spread it into a two-dimensional laser light sheet. In the plane of the laser light sheet, the flow was visualized by means of tracer ink (Rhodamine B and poster paints (turquoise, fluorescent pink, fluorescent green and fluorescent orange) with same specific gravity of water), which oozed out from two dye ports at plus-minus 60 degree from the front stagnation point of the circular cylinder. The visualized flow patterns were monitored by the CCD video camera and recorded on video recorder.

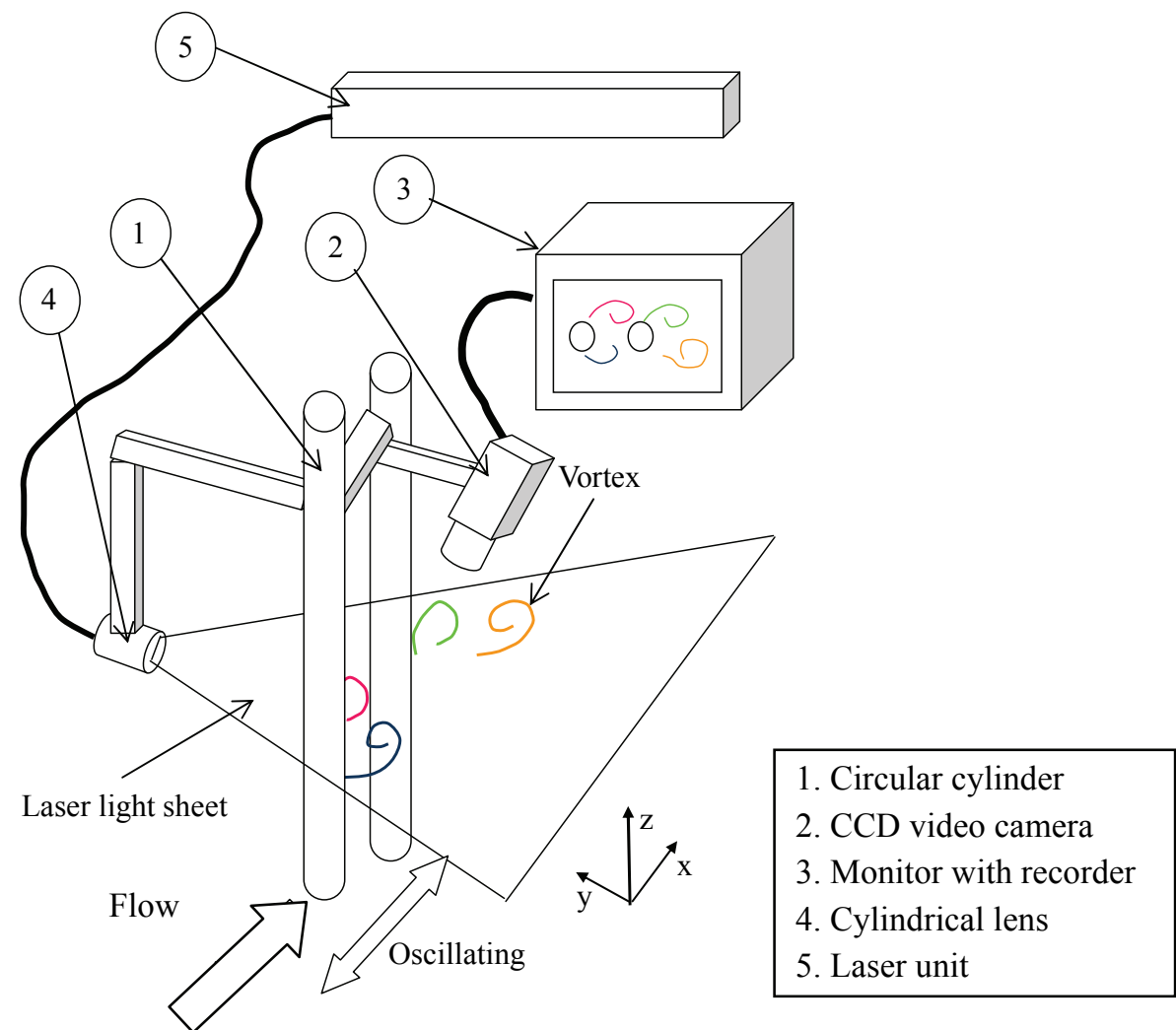

Fig. 5 Schematic diagram of the layout of flow visual apparatus. The camera is attached so that it may move with movement of the circular cylinder. The laser light sheet is not used for photography using poster paint.

\subsection{Experimental parameters}

The main experimental parameters given by the distance ratio $L / d$ (the ratio of cylinder distance $L$ to the outside diameter of cylinder $d$ ), the arrangement angle $\alpha$ (angle with the line which connects two circular cylinder center to flow) the oscillation frequency ratio $f / f_{K}$ (the ratio of circular cylinder oscillation frequency $f$ to natural Karman vortex's frequency $f_{K}$ ), the amplitude ratio $2 a / d$ (the ratio of half amplitude of cylinder motion $a$ to the outside diameter of cylinder $d$ ) and the main flow velocity in the test section $U$. The distance ratio $L / d$ was changed three stage $(L / d=1.5$, 2.5 and 5.5). It is known that three kinds of these distance ratio $L / d$ shows a characteristic flow pattern in two circular cylinders of tandem arrangement or parallel arrangement (JSME Standard, 1998). The arrangement angle $\alpha$ is seven kinds, 0 degree (tandem arrangement), 15 degrees, 30 degrees, 45 degrees, 60 degrees, 75 degrees, and 90 degrees (parallel arrangement). The oscillation frequency ratio $f / f_{K}$ was varied from 0.0 to 7 (28 steps), and the amplitude ratio 
$2 a / d$ was set $0.25,0.50,0.75$ and 1.0 . The main flow velocity $U$ was $0.041 \mathrm{~m} / \mathrm{s}$ which correspond to Reynolds number about $640(\operatorname{Re}=U d / v$, where $v$ is the kinematic viscosity of water).

\subsection{Experimental procedure}

As preparation, the flow velocity of the test section is set up by inputting operation frequency into the operator control panel of the closed circuit water channel. One circular cylinder is installed into an aimed flow and tracer ink is oozed. The tracer ink was oozed into the flow from two dye ports on a stationary circular cylinder and the visualized aspects of separated wake flow were recorded on video tapes. The natural Karman vortex shedding frequency $f_{K}$ was measured by counting the visualized vortices shed from the stationary circular cylinder for a certain period of time. (In this experiment, the value of Karman vortex frequency $f_{K}$ was $0.45 \mathrm{~Hz}$.)

In the experiment of two steady circular cylinders, after setting up the distance ratio $L / d$ and the arrangement angle $\alpha$, the tracer ink is made to ooze from each circular cylinder, and each vortex flow is observed. In the experiment of two in-line oscillating circular cylinders, after carrying out the setup of cylinder distance $L$, and the setup of the arrangement angle $\alpha$, the setup of the oscillating amplitude $a$ and the setup of the oscillating frequency $f$ were performed. Here, the cylinder distance $L$, the half-amplitude of oscillation $a$ and the cylinder oscillation frequency $f$ were set to be desired values of the distance ratio $L / d$, the amplitude ratio $2 a / d$ and the oscillation frequency ratio $f / f_{K}$. The tracer ink was oozed and the visualized flow feature from the oscillating cylinders was monitored and recorded on video recorder. The vortex shedding frequency $f_{V K}$ was obtained from the number and the measurement time of past vortex at the observation point. The cylinder oscillation frequency $f$ was calculated by measuring an oscillation cycle. The measurement technique of vortex shedding frequency is the primitive technique which plays video and is performed by viewing of man. Neither image-processing equipment nor image-processing software is used for the measurement. Refer to the reference (Yokoi and Hirao, 2008) for the description about the measurement accuracy and error. The value of the standard deviation of the Strouhal number obtained in this experiment was 0.004.

\section{Experimental results and discussion}

\subsection{Vortex shedding characteristics in the steady setting case}

Figure 6 shows the variation of the Strouhal number $S t$ for every arrangement angle $\alpha$ in each distance ratio $L / d$. Here, the Strouhal number is defined by $S t=f_{K} d / U$. The $f_{K}$ is time mean vortex shedding frequency form stationary cylinder. In the figure, the abscissa is the arrangement angle $\alpha$ and the ordinate is the Strouhal number St. All over the figure, the closed symbol shows the 1st circular cylinder, and the open symbol shows the 2nd circular cylinder. In distance ratio $L / d=5.5$, and 2.5 and 1.5, the arrangement angles whose gap is lost in projection of two circular cylinders were 10.47 degrees, 23.57 degrees, and 41.81 degrees, respectively. And the characteristic flow patterns of two circular cylinders of staggered arrangement are shown in Fig. 7. In the Fig. 6, the result of the case where the circular cylinder interval is wide $(L / d=5.5)$ is shown by the circle symbol (red line). There is almost no difference of the 1 st cylinder and the 2 nd cylinder. And if the arrangement angle exceeds 30 degrees, it can be seen that it is not different from the case of an independent circular cylinder. When the distance ratio became small, by the case where there is the vortex shedding, the difference occurred from the 1 st circular cylinder to vortex shedding frequency and from the 2 nd circular cylinder to vortex shedding frequency, and even if it moreover averaged the two values, it resulted in keeping away from the value of the vortex shedding frequency of the single circular cylinder. In the case of distance ratio $L / d=5.5$, it was the case of the arrangement angle being 45 degrees that whose average value of the Strouhal number of two circular cylinders was the largest, and the value was 0.205 . In the case of distance ratio $L / d=2.5$, it was the case of the arrangement angle being 30 degrees that whose average value of the Strouhal number of two circular cylinders was the largest, and the value was 0.271 . When an arrangement angle is 45 degrees, the average value of the Strouhal number of two circular cylinders is 0.267 , and differs from the value in the case of single circular cylinder greatly. In the case of distance ratio $L / d=1.5$, it was the case of an arrangement angle being 75 degrees that whose average value of the Strouhal number of two circular cylinders was the largest, and the value was 0.239 . When an arrangement angle is 45 degrees, since the vortex shedding from the 1 st circular cylinder is not observed, the average value is not calculated. It can grasp that the state where the mutual interference of two circular cylinders is observed notably is the case where the 


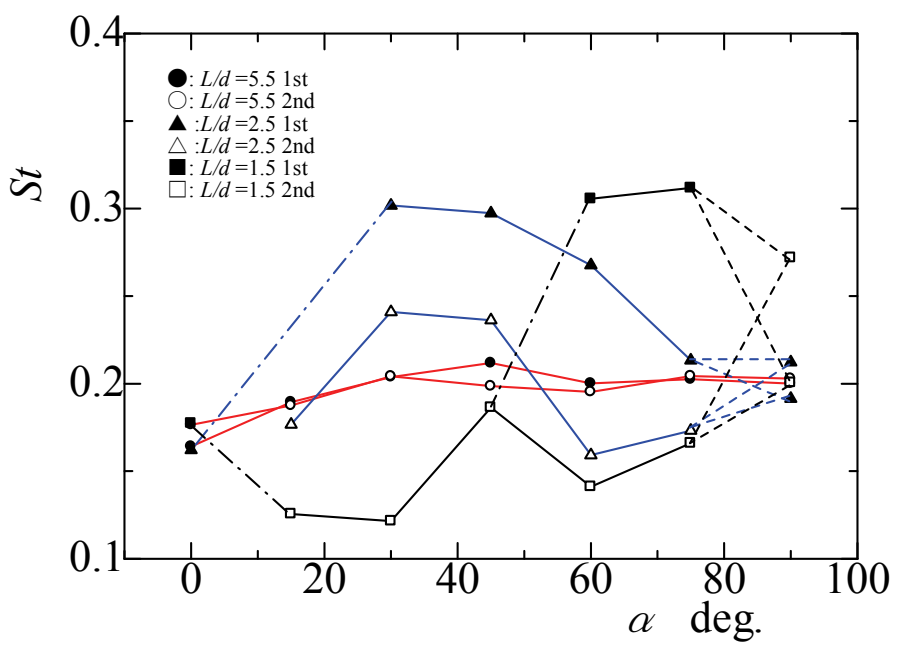

Fig. 6 Variation of vortex shedding frequency from stationary two cylinders in the case of each distance ratio $L / d$. The closed symbol shows the 1 st circular cylinder, and the open symbol shows the 2 nd circular cylinder. The arrangement angles whose gap is lost in projection of two circular cylinders were 10.47 degrees, 23.57 degrees, and 41.81 degrees, respectively.
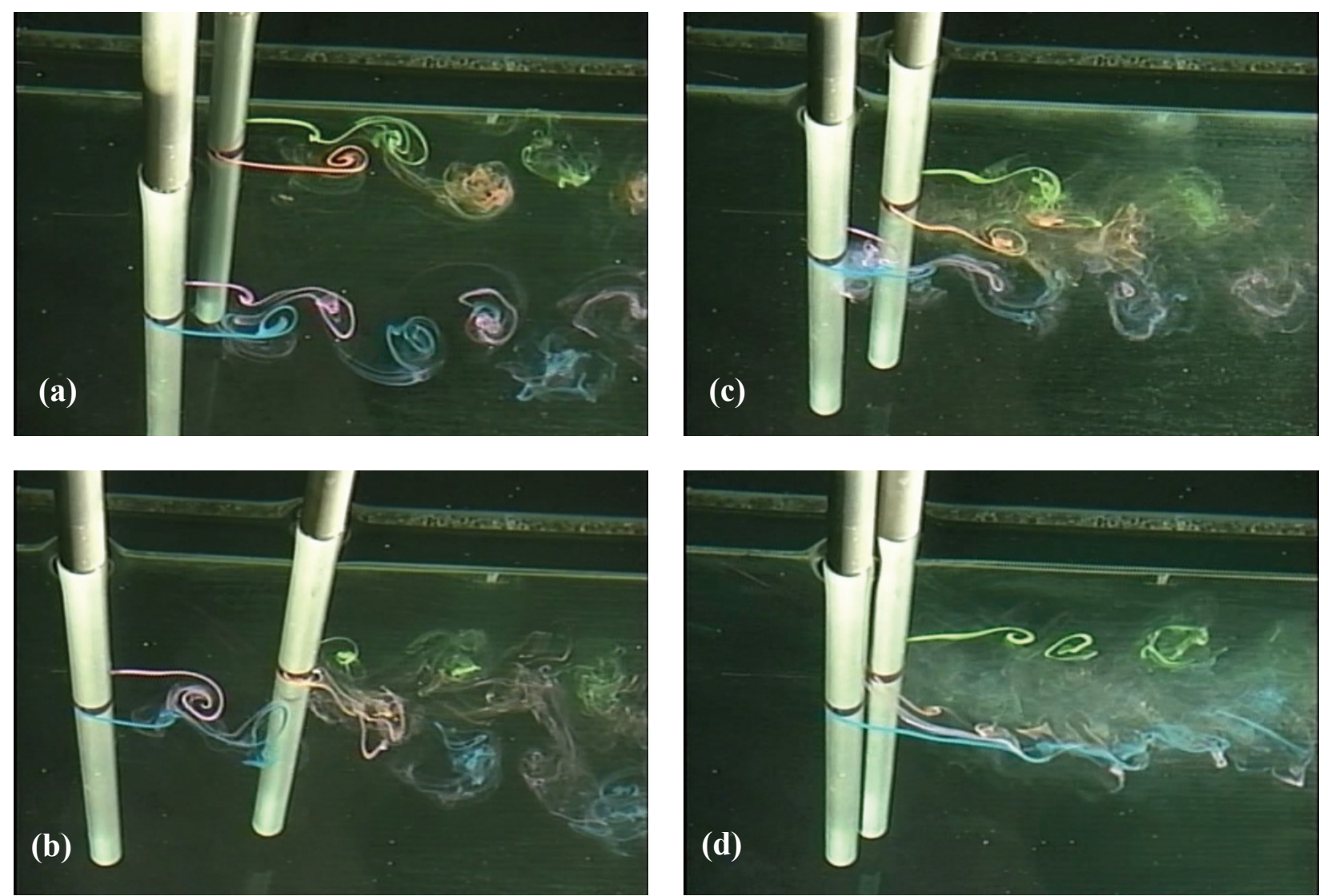

Fig. 7 Characteristic flow patterns of two circular cylinders of staggered arrangement; (a) $L / d=5.5, \alpha=75$ degrees, (b) $L / d=5.5, \alpha=15$ degrees, (c) $L / d=2.5, \alpha=45$ degrees, (d) $L / d=1.5, \alpha=45$ degrees. The main flow is flowing into the right from the left. 
distance ratio is $L / d=2.5$. Figure 7 (a) shows the flow pattern in the state where the arrangement angle is 75 degrees. The Karman vortex street which became independent like the time of an independent circular cylinder can be seen discharged from each circular cylinder. Figure 7 (b) shows the flow pattern in the state where an arrangement angle is 15 degrees. The vortex discharged from the inner side of the 1 st cylinder contacts inside the 2 nd cylinder, and an aspect that the vortex discharged from the inner side of the 2nd cylinder and the vortex discharged from the inner side of the 1 st cylinder synchronize can be seen.

If the interval of the circular cylinder narrows, a variation will begin to appear in a vortex shedding from each circular cylinder. According to the visualization photograph of Ishigami et al. (1972), the width of the wake of the circular cylinder in the upstream side becomes narrow, and the width of the wake of the circular cylinder in the downstream side becomes wide. Accordingly, from the 1st circular cylinder, large vortices are discharged with a long time interval, and small vortices are discharged with a short time interval from the 2nd circular cylinder. So, a difference will occur in the vortex shedding frequency of two circular cylinders. Figure 7 (c) shows the flow pattern in the state where the arrangement angle is 45 degrees in distance ratio $L / d=2.5$. It shows clearly the difference in wake width, and the difference in the vortex roll-up position. At the 1st cylinder, the vortex is formed near the circular cylinder and its wake width becomes narrow. On the other hand, at the 2nd cylinder, the vortex is formed in the position distant from the circular cylinder, and its wake width becomes wide. The result of the case of distance ratio $L / d=2.5$ is shown by the triangle symbol (blue line) in Fig. 6. Here, since in the case of $\alpha=0$ degree (tandem arrangement) arrangement angle the separating shear layer from the 1 st cylinder adhered to the 2 nd cylinder, or had covered and hung and the 2 nd cylinder was placed into the stagnation region of the 1 st cylinder behind, there was no vortex shedding from the 2 nd cylinder. Since the separating shear layer inside the 1 st cylinder had collided with the 2 nd cylinder when the arrangement angle was 15 degrees, the vortex shedding from the 1st cylinder did not grow into the alternate vortex shedding. In the arrangement angle of 60 degrees, and 75 degrees, when the value of the Strouhal number of the 1 st cylinder and the value of the Strouhal number of the 2 nd cylinder are averaged, there is no change so much with the case of an independent circular cylinder. However, at the arrangement angle of 30 degrees, and 45 degrees, since it differs from the case of an independent circular cylinder even if it averages the value of the Strouhal number, it is guessed that these arrangement angles are carrying out mutual interference most.

Furthermore, when the circular cylinder interval narrowed more, the aspect of vortex shedding became complicated, and the mutual interference appeared notably. Figure 7 (d) shows the flow pattern in the state where the arrangement angle is 45 degrees in distance ratio $L / d=1.5$. Since each circular cylinder is close, the aspect of the flow is very complicated. The result in distance ratio $L / d=1.5$ is shown to Fig. 6 by the square symbol (black line). In the case where the arrangement angle is 0 degree (tandem arrangement), the separating shear layer from the 1 st cylinder has been rolled behind the 2 nd cylinder. Therefore, there is no vortex shedding from the 2 nd cylinder. In the range of the arrangement angle from 15 to 45 degrees, the shear layer which separates from the 1 st cylinder flows so that it may meet with the shear layer which separates from the inner side of 2 nd cylinder. And the union vortex of a size which balances with the vortex of the separating shear layer of the outside of the 2 nd cylinder is formed. Here, the vortex shedding from the 1 st cylinder was not confirmed. When arrangement angles are 60 degrees and 75 degrees, the vortex had been whirled around near the 1st cylinder interferes with roll up of the separating shear layer inside the 2 nd cylinder. Therefore, the wake width of the 1st cylinder becomes narrow, and the wake width of the 2nd cylinder becomes also wide. According to this phenomenon, with the 1st cylinder, the vortex shedding frequency becomes high, and the vortex shedding frequency becomes low with the 2 nd cylinder. In the case of distance ratio $L / d=1.5$, it was found that mutual interference occurs in the range of all arrangement angles.

\subsection{Vortex shedding characteristics in the oscillating case}

It is one of the most interesting things in this study to investigate the mutual interference of the two circular cylinder wakes in an unsteady flow. The arrangement angle of two circular cylinders was made into 45 degrees from the experimental result in steady flow, the distance ratio $L / d$, the amplitude ratio $2 a / d$, and the oscillation frequency ratio $f / f_{K}$ were varied, and experiment investigation was performed. The variation of the vortex shedding frequency by circular cylinder oscillation is shown in Figs. 8 10. Here, Figs. 8, 9, and 10 show the case of the distance ratio $L / d=1.5$, the distance ratio $L / d=2.5$, and the distance ratio $L / d=5.5$, respectively. In those figures, the abscissa is the oscillation frequency ratio $f / f_{K}$ and the ordinate is the vortex shedding frequency ratio $f_{V K} / f_{K}$. Two solid lines in the figure have the 
ratio of the vortex shedding frequency in case of the cylinder oscillation $f_{V K}$ and the circular cylinder oscillating frequency $f$ and mean the "lock-in" status. Therefore, the occurrence of lock-in can be assumed when experiment data are shown on the line. Those dashed lines mean the vortex shedding frequency from each circular cylinder when the circular cylinder is not oscillating. The red dashed line and the blue dashed line show the vortex shedding frequency of the 1 st cylinder in stationary case, and the vortex shedding frequency of the 2nd cylinder in stationary case, respectively. In those figures, circle symbol is the experimental result of the 1st cylinder, and square symbol is the experimental result of the 2 nd cylinder. The lock-in was seen even if the mutual interference of two circular cylinders was in the state of appearing strongly. Although the range of the oscillation frequency which carries out the lock-in also to the 1 st cylinder and the 2 nd cylinder was about the same in the high oscillation frequency side, differing was found with the 1st cylinder and the 2nd cylinder at the low oscillation frequency side. If a distance ratio is small, it will be found that the oscillation frequency range in which the 2nd circular cylinder carries out a lock-in is wider than the 1 st circular cylinder. Figure 8 shows the case where a distance ratio is the smallest. Here, from the 1st circular cylinder, although the vortex shedding frequency at the time of circular cylinder stationary is shown by the dotted line all over each figure, since there is no vortex shedding, only the 2nd circular cylinder is shown. The Fig. 8(a) is the case that an oscillating amplitude ratio is the smallest, and it can be found with the 1st circular cylinder and the 2nd circular cylinder that the range and form of a lock-in differ from each other. The maximum oscillating frequency ratio which a lock-in produces was $f / f_{K}=5.7$. If the oscillating amplitude ratio is enlarged, the range and form of the lock-in can be seen become the same. However, the values of the maximum oscillating frequency ratio which the lock-in produces are low. The maximum oscillating frequency ratio which the lock-in produces was $f / f_{K}=4$ at the time of oscillating amplitude ratio $2 a / d=0.5$ as shown in the Fig. $8(\mathrm{~b})$. Similarly, the maximum oscillating frequency ratios which the lock-in produces from the Figs. $8(\mathrm{c})$ and $8(\mathrm{~d})$ were $f / f_{K}=3.9$ and $f / f_{K}=3.3$, respectively. Figure 9 shows the case where the distance ratio is $L / d=2.5$. The same tendency as the case of distance ratio $L / d=1.5$ can be seen. The value of the maximum oscillating frequency ratio which the lock-in produces became small when the oscillating amplitude ratio became large. The value of the maximum oscillating frequency ratio can be read with $f / f_{K}=5.4$ by the Fig. 9 (a), can be read with $f / f_{K}=4$ by the Fig. $9\left(\right.$ b), and can be read with $f / f_{K}=3.5$ by the Fig $9(\mathrm{c})$, and can be read with $f / f_{K}=3$ by the Fig. $9(\mathrm{~d})$. Figure 10 shows the case where the distance ratio is large. Except for the case where it is $2 a / d=0.25$ with the smallest value of the oscillating amplitude ratio, the vortex shedding from the 2 nd circular cylinder was the same as the vortex shedding from the 1 st circular cylinder mostly. The value of the maximum oscillating frequency ratio which the

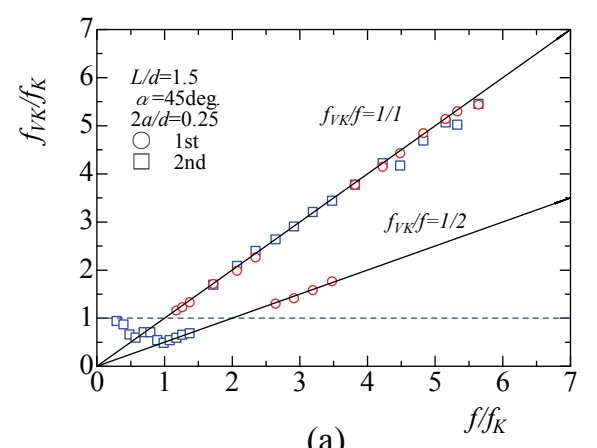

(a)

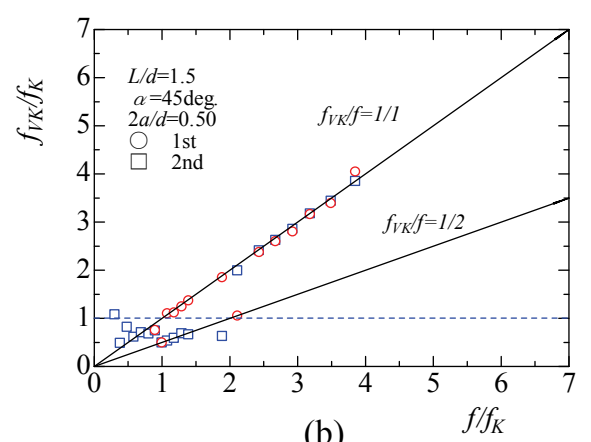

(b)

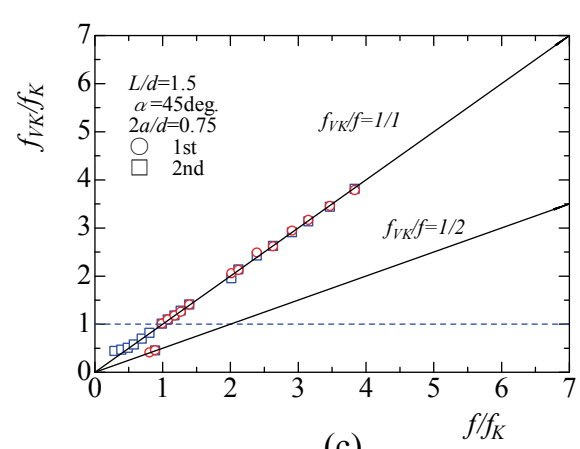

(c)

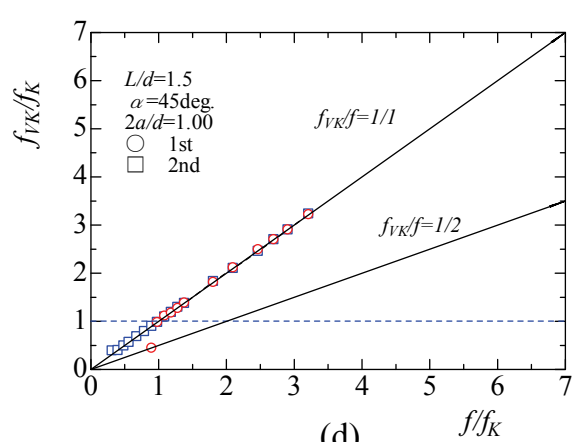

(d)

Fig. 8 Variation of vortex shedding frequency in the case of $L / d=1.5$ and $\alpha=45$ degrees; (a) $2 a / d=0.25$, (b) $2 a / d=0.50$, (c) $2 a / d=0.75$, (d) $2 a / d=1.00$ 


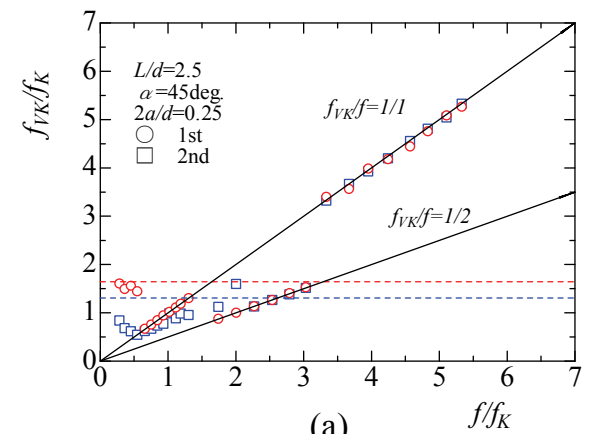

(a)

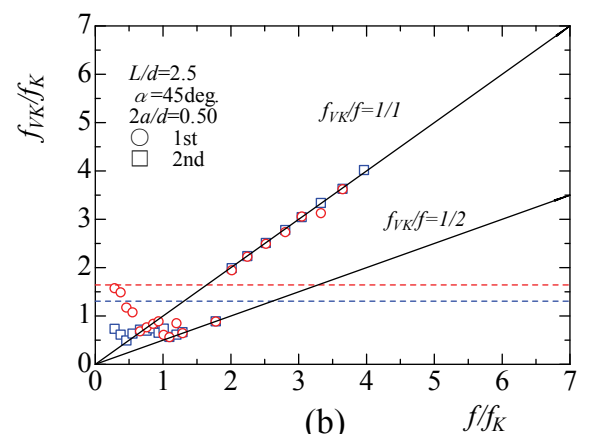

(b)

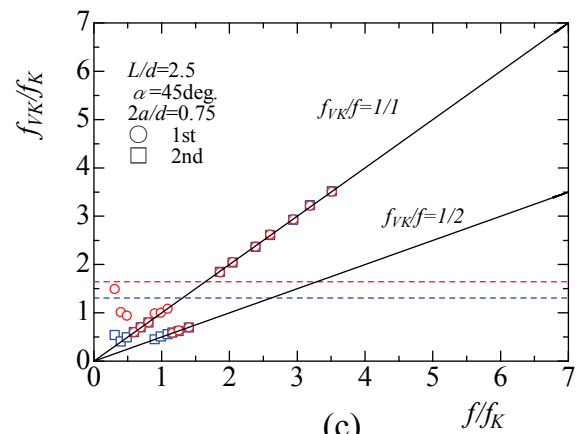

(c)

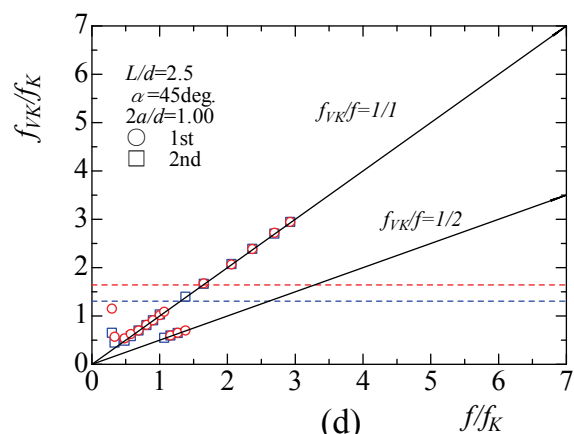

(d)

Fig. 9 Variation of vortex shedding frequency in the case of $L / d=2.5$ and $\alpha=45$ degrees; (a) $2 a / d=0.25$, (b) $2 a / d=0.50$, (c) $2 a / d=0.75$, (d) $2 a / d=1.00$
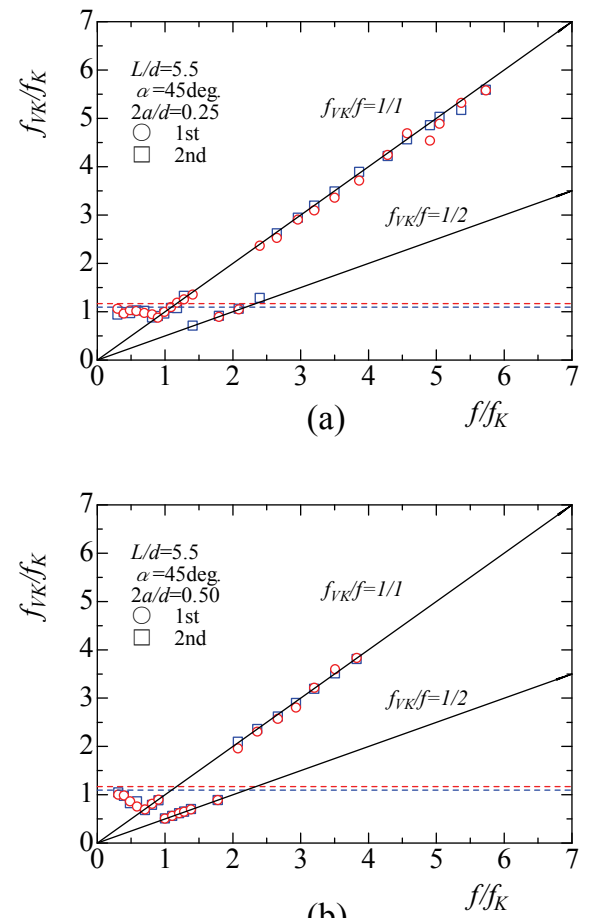

(b)

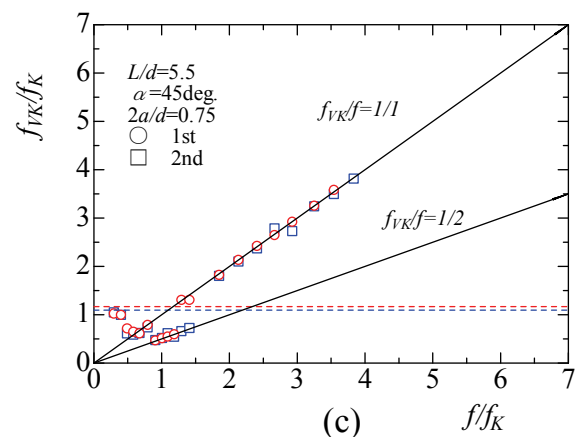

(c)

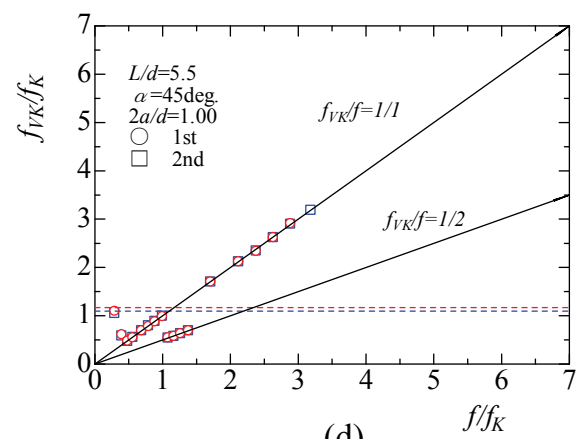

(d)

Fig. 10 Variation of vortex shedding frequency in the case of $L / d=5.5$ and $\alpha=45$ degrees; (a) $2 a / d=0.25$, (b) $2 a / d=0.50$, (c) $2 a / d=0.75$, (d) $2 a / d=1.00$ 

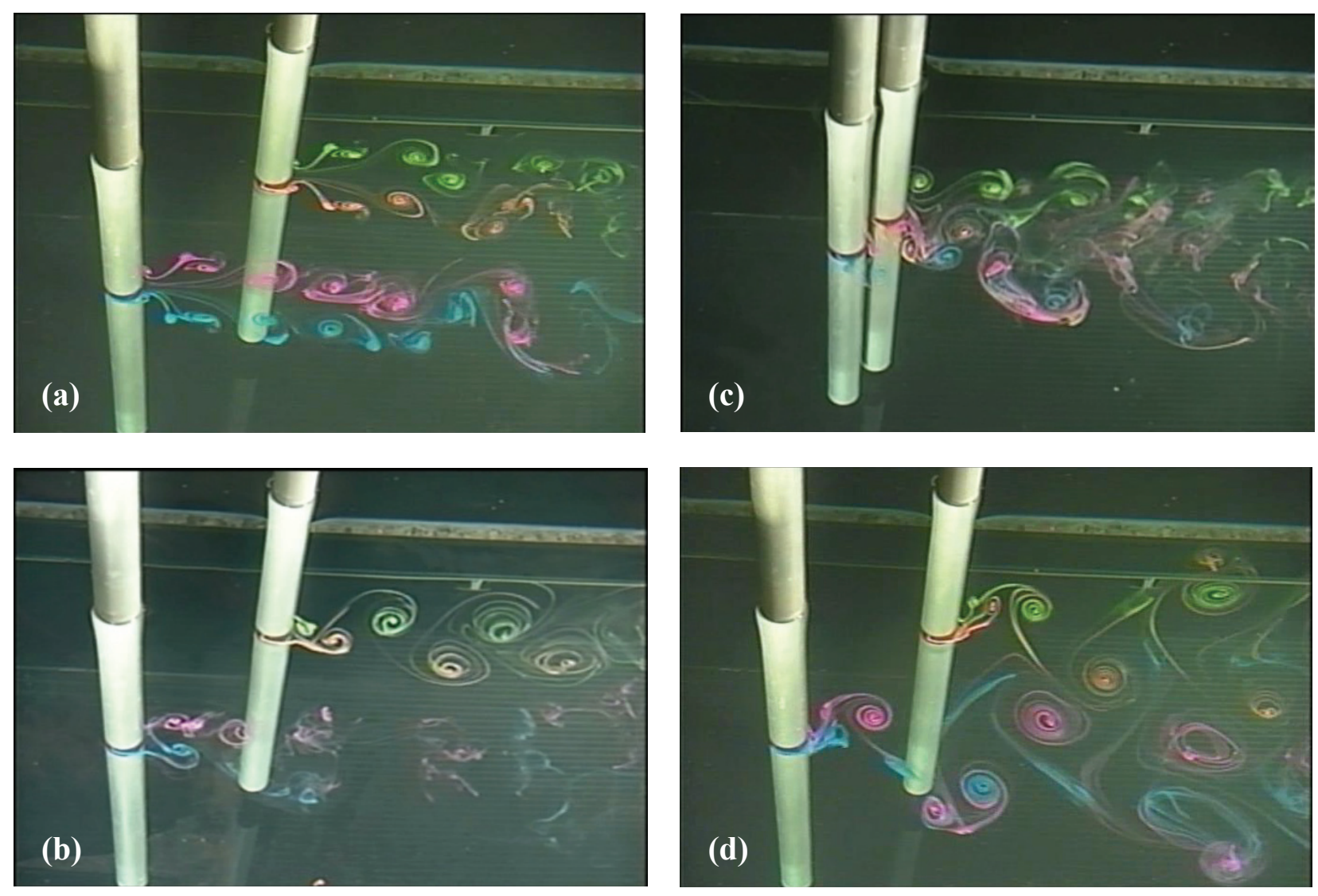

Fig. 11 Characteristic flow patterns of two circular cylinders of staggered arrangement at the cylinder oscillation case; (a) $L / d=5.5,2 a / d=0.50, f / f_{K}=2.1$, (b) $L / d=5.5,2 a / d=0.25, f / f_{K}=2.4$, (c) $L / d=1.5,2 a / d=0.50, f / f_{K}=2.1$, (d) $L / d=5.5,2 a / d=0.50, f / f_{K}=1.8$

lock-in produces became small similarly, when the oscillating amplitude ratio became large. The value of the maximum oscillating frequency ratio can be read with $f / f_{K}=5.7$ by the Fig. $10(\mathrm{a})$, can be read with $f / f_{K}=3.9$ by the Fig. $10(\mathrm{~b})$, can be read with $f / f_{K}=3.8$ by the Fig. $10(\mathrm{c})$, and can be read with $f / f_{K}=3.2$ by the Fig. $10(\mathrm{~d})$. Regardless of the magnitude of the distance ratio, when the oscillation amplitude was increased, it was found that the lock-in range tends to narrow.

Four characteristic flow patterns at the time of lock-in were obtained by having oscillated two circular cylinders of staggered arrangement in the direction of the flow. Those flow patterns are shown in Fig. 11. The case where the simultaneous vortex shedding lock-in of both of circular cylinders is being carried out is shown in Fig. 11(a). Two twin vortex streets which became independent mutually to the pair of circular-cylinder wake can be seen. The flow patterns with which the simultaneous vortex shedding lock-in and the alternate vortex shedding lock-in combined are shown in Figs. 11(b) and 11(c). In Fig. 11(b), the 1st cylinder shows the simultaneous vortex shedding lock-in, and the 2nd cylinder shows the alternate vortex shedding lock-in. On the other hand, in Fig, 11(c), it is the opposite combination. The 1st cylinder shows the alternate vortex shedding lock-in, and the 2nd cylinder shows the simultaneous vortex shedding lock-in. The case where the alternate vortex shedding lock-in of both of circular cylinders is being carried out is shown in Fig. 11(d). The pair vortex of mushroom section shape is discharged from both of circular cylinders, and one pair of alternate vortex streets is formed.

\section{Conclusion}

The aspects of the vortex shedding characteristic and the flow features were observed by the visualization experiment to which the interval and arrangement angle of two circular cylinders were varied. The following conclusions were obtained. 
(1) Each vortex shedding characteristic of two circular cylinders changes with arrangement angles, and if the distance ratio is small, mutual interference will become remarkable, and the difference between the 1st circular cylinder and the 2 nd circular cylinder becomes remarkable.

(2) Even if the mutual interference flow had occurred, when the circular cylinder was oscillated, it was found that the lock-in phenomenon occurs with each circular cylinder. The lock-in range and the lock-in type were shown for every distance ratio. It was obtained when the oscillating amplitude was increased, the lock-in range tends to narrow.

(3) Four kinds of typical flow patterns at the time of the lock-in of staggered arrangement oscillating two circular cylinders were shown.

\section{Acknowledgment}

The authors would like to express their deep thanks to Mr Hiroya Komatsu, Mr Satoshi Mizuno and Mr Ken Kawano for their contribution to experimental research on this work.

\section{References}

Chen, S. S., A review of flow-induced vibration of two circular cylinders in crossflow, Transactions of the ASME J Pressure Vessel Technology, Vol. 108, No. 4 (1986), pp. 382-393.

Ishigami, S., Nishikawa, E., Nishimura, K., and Cho, K., Experimental Study on Structure of Gas Flow in Tube Banks with Tube Axes Normal to Flow, Bull. JSME, 15 (1972), pp.949-956 (in Japanese).

Kim, S. and Sakamoto, H., A Study on Characteristics of Flow-Induced Vibrations of Two Circular Cylinders in Staggered Arrangement, Transactions of Japan Society of Mechanical Engineers, Series B, Vol.73, No.725(2007), pp.139-146 (in Japanese).

Sakamoto, H., Alam, Md. M., Kim, S. and Takai, K., A Study on Flow Around Two Circular Cylinders in Staggered Arrangements (1st Report, Characteristics of Fluid Forces), Transactions of Japan Society of Mechanical Engineers, Series B, Vol.70, No.696(2004a), pp.2034-2042 (in Japanese).

Sakamoto, H., Alam, Md. M., Kim, S. and Takai, K., A Study on Flow Around Two Circular Cylinders in Staggered Arrangements (2nd Report, Estimation of Strouhal Number Using in Combination with Wavelet Analysis), Transactions of Japan Society of Mechanical Engineers, Series B, Vol.70, No.699(2004b), pp.2830-2837 (in Japanese).

Sarpkaya, T. and Cinar, M., Hydrodynamic interference of two cylinders in harmonic flow, Proceedings of Annual Offshore Technology Conference, Vol. 12, No. 2(1980), pp. 333-340.

Sumner, D., Price, S. J. and Paidoussis, M. P., Flow-pattern identfication for two staggered circular cylinder, Journal of Fluid Mechanics, Vol. 411 (2000), pp. 263-303.

The Japan Society of Mechanical Engineers ed., JSME Standard: Guideline for Evaluation of Flow-Induced Vibration of a Cylindrical Structure in a Pipe (1998), The Japan Society of Mechanical Engineers (in Japanese).

Williamson, C. H. K., Sinusoidal flow relative to circular cylinders, Journal of Fluid Mechanics, Vol. 155 (1985), pp. 141-174.

Yokoi, Y. and Hirao, K., The fundamental study about flow visualized observation by use of a towing water tank; vortex shedding from a bluff cylinder, Transactions of the Visualization Society of Japan, Vol. 28, No. 6(2008), pp.33-37 (in Japanese).

Zdravkovich, M. M., Flow Around Circular Cylinder, Vol.1 Fundmentals (1997), Oxford University Press.

Zdravkovich, M. M., Flow Around Circular Cylinder, Vol.2 Applications (2003), Oxford University Press. 